\title{
Synthesis and Characterization of an Iron Nitride Constructed by a Novel Template of Metal Organic Framework
}

\author{
Suyan Liu, ${ }^{1,2}$ Quan Huo, ${ }^{1}$ Rongna Chen, ${ }^{1}$ Peipei Chen, ${ }^{1}$ Yuan Li, ${ }^{1}$ and Yang Han ${ }^{2}$ \\ ${ }^{1}$ Hebei Key Laboratory of Applied Chemistry, College of Environmental and Chemical Engineering, Yanshan University, \\ Qinhuangdao, Hebei 066004, China \\ ${ }^{2}$ The State Key Laboratory of Heavy Oil Processing, Department of Materials Science and Engineering, China University of Petroleum, \\ Beijing, Changping District, Beijing 102249, China
}

Correspondence should be addressed to Quan Huo; quanhuo@ysu.edu.cn

Received 31 July 2014; Accepted 27 November 2014

Academic Editor: Bing Wu

Copyright (C) 2015 Suyan Liu et al. This is an open access article distributed under the Creative Commons Attribution License, which permits unrestricted use, distribution, and reproduction in any medium, provided the original work is properly cited.

\begin{abstract}
An iron nitride with high surface area was synthesized from an iron-based metal organic framework (Fe-MOF) in this work. During the synthesis process, the Fe-MOF of MIL-53 served as a hard template, a template to impart a certain degree of morphology for iron oxide products and to form porosities for iron nitride products. Moreover, it played the roles of iron sources for the synthesis of the final iron oxides and the iron nitrides. The physicochemical properties of the materials were characterized by a series of technologies including XRD, SEM, and $\mathrm{N}_{2}$-adsorption/desorption. The results showed that the iron nitride synthesized from MIL53 was $\alpha-\mathrm{Fe}_{2-3} \mathrm{~N}$. And, the $\alpha-\mathrm{Fe}_{2-3} \mathrm{~N}$ showed the morphology with loosely aggregated particles which favored the formation of rich interparticle porosities. As a result, the surface area of the $\alpha-\mathrm{Fe}_{2-3} \mathrm{~N}$ was larger than those of samples using $\alpha-\mathrm{Fe}_{2} \mathrm{O}_{3}$ as precursors and its value was $41 \mathrm{~m}^{2} / \mathrm{g}$. In addition, the results agreed that both raw material properties (such as crystallinity and surface areas) and nitriding approaches had significant effects on the surface areas of iron nitrides. Also the results were proved by the iron oxide synthesized with different methods. This new synthetic strategy could be a general approach for the preparation of late transition metal nitrides with peculiar properties.
\end{abstract}

\section{Introduction}

Transition metal nitride catalysts have attracted great attentions because they exhibit noble metal-like characteristics in many reactions, such as fuel cells, optical coatings, electrical contacts, and catalysts [1]. The key to producing successful catalysts lies in the ability to synthesize materials of high surface areas [2]. Synthetic strategies of transition metal nitrides with high surface areas have been intensively studied, including temperature-programmed reaction of solidstate metal compounds with gaseous nitrogen sources [36], pyrolysis of metal precursors [7], and solution reactions [8] as well as developed reactive hard template [9] and soft urea pathway [10]. Recently, Yang and DiSalvo [11] reported a simple process prepared mesoporous transition metal nitrides ( $\mathrm{NbN}, \mathrm{VN}, \mathrm{Ta}_{3} \mathrm{~N}_{5}$, and TiN) by the ammonolysis of bulk ternary oxides that contain cadmium. Depending upon the starting composition and the ammonolysis temperature, the TMNs had pore sizes from 10 to $40 \mathrm{~nm}$, domain sizes of $5-40 \mathrm{~nm}$, and the surface areas $15-60 \mathrm{~m}^{2} / \mathrm{g}$. However, most of the nitrides are early transition metal nitrides focusing on nitrides of molybdenum and tungsten, and only a few papers have reported the synthesis of late transition metal nitrides particles such as iron nitrides, due to their relatively low thermal stability $[8,12-15]$. For example, Koltypin et al. [12] reported the sonochemical synthesis of iron nitride nanoparticles by two methods, that is, sonication of $\mathrm{Fe}(\mathrm{CO})_{5}$ in a decane solution under a gaseous $\mathrm{NH}_{3} / \mathrm{H}_{2}$ mixture and nitridation of sonochemically prepared amorphous iron under a mixed $\mathrm{NH}_{3} / \mathrm{H}_{2}$ stream. Later on, iron particles of various grain sizes [13], amorphous iron prepared through reducing ferrous sulfate by potassium borohydride [14], and $\mathrm{Fe}_{2} \mathrm{O}_{3}$ [15] were also used as precursor to produce iron nitride by a temperature-programmed reaction in $\mathrm{NH}_{3}$. 
Recently, Choi and Gillan [8] reported the use of solvothermally moderated metal azide decomposition as a route to nanocrystalline mid to late transition metal nitrides. This method utilizes exothermic solid-state metathesis reaction precursor pairs, namely, metal halides $\left(\mathrm{NiBr}_{2}, \mathrm{FeCl}_{3}\right.$, and $\mathrm{MnCl}_{2}$ ) and sodium azide, but conducts the metathesis reaction and azide decomposition in superheated toluene. The reaction temperatures are relatively low $\left(<300^{\circ} \mathrm{C}\right)$ and yield thermally metastable nanocrystalline hexagonal $\mathrm{Ni}_{3} \mathrm{~N}$ and $\mathrm{Fe}_{2} \mathrm{~N}$ and tetragonal $\mathrm{MnN}$. Moszyński et al. [16] reported that three iron samples of various mean sizes of crystallites were nitrided in gas mixtures with $\mathrm{NH}_{3} / \mathrm{H}_{2}$ ratio increasing stepwise up to the pure ammonia. The higher the mean size of crystallites was, the lower nitriding potential was required to initiate the phase transition $\alpha$ - $\mathrm{Fe} \rightarrow \gamma$ - $\mathrm{Fe}_{4} \mathrm{~N}$ observed during the nitriding process. Unfortunately, the surface areas of these iron nitrides are usually lower than $40 \mathrm{~m}^{2} / \mathrm{g}$. In order to produce promising iron nitride catalysts, new ways to synthesize iron nitrides with higher surface areas are thus in great demand.

Metal organic frameworks (MOFs) as a kind of emerging nanoporous material, built from connectors and organic linkers, have attracted many attentions due to their intriguing architectures and topologies, tunable pore sizes, and wide potential applications such as gas storage and separation and drug delivery [17]. The various pore sizes, high thermal stability, and synthetically flexible environment of MOFs make them feasible as templates for preparing nanoporous carbon materials [18], nanoscale $\mathrm{NaAlH}_{4}$ [19], transition metal nanoparticles [20], silver cluster [21], and so forth. Recently, Kim et al. [22] reported a versatile method to prepare metal nanoparticles supported on nanoporous carbon (M/NC3) via carbonization and carbothermal reduction (CCR) of metalcoordinated IRMOF-3 materials by postsynthetic modification (PSM) with metal precursors (i.e., $\mathrm{Ru}, \mathrm{W}, \mathrm{V}$, and $\mathrm{Ti}$ ). Use of IRMOF materials as templates/carbon sources led to desirable pore characteristics in the resulting materials, including high surface area $\left(900-2000 \mathrm{~m}^{2} / \mathrm{g}\right)$ coupled with an increased mesoporosity $\left(0.14-0.34 V_{\text {micro }} / V_{\text {pore }}\right)$. However, examples of metal organic framework as hard template to synthesis of iron nitride are rare. Transition metal ions are usually used as connectors; thus, MOFs provide the possibility to be a porous reactive hard template candidate. As a typical example of Fe-MOFs, the common MIL-53(Fe) is chosen, which is built from infinite chains of corner sharing $\mathrm{Fe}^{\mathrm{III}}{ }_{4}(\mathrm{OH})_{2}$ octahedra interconnected by dicarboxylate groups of benzene dicarboxylate units [23]. In this way, a 3D microporous framework with $1 \mathrm{D}$ diamond shaped channels with free internal diameter of about $0.85 \mathrm{~nm}$ is formed.

Herein, we report the synthesis of iron nitride from ironbased metal organic framework exemplified by octahedral MIL-53(Fe) not only as a reactive hard template but also as a provider of $\mathrm{Fe}$ source. The difference in BET surface area of iron nitride between MOFs and $\mathrm{Fe}_{2} \mathrm{O}_{3}$ is of interest. For this purpose, $\mathrm{Fe}_{2} \mathrm{O}_{3}$ is synthesized by the sol-gel method and MOFs as template ways [24] according to the literature, respectively. All of the $\mathrm{Fe}_{2} \mathrm{O}_{3}$ and MIL-53 were employed to prepare iron nitride and their BET surface areas were

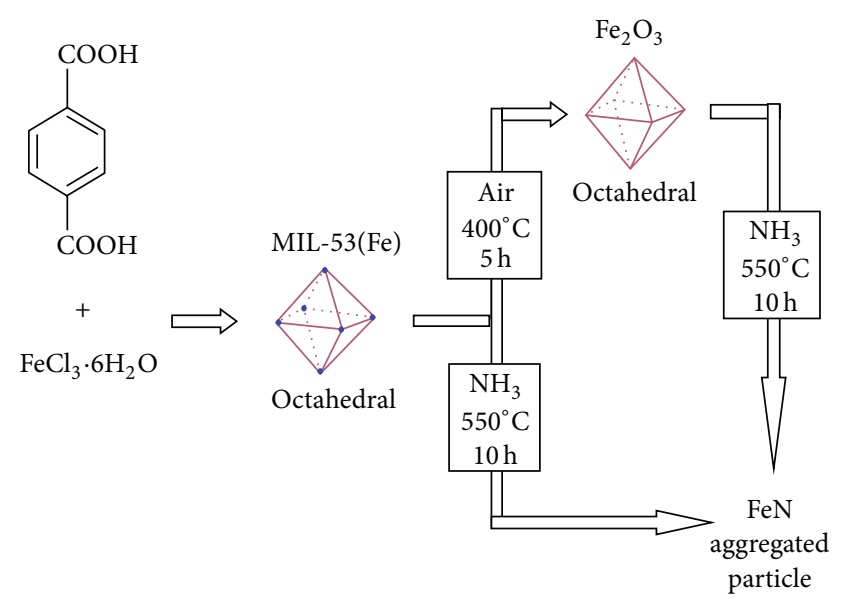

Scheme 1: Selective preparation routes of iron oxide and iron nitride using MOFs as a reactive template.

analyzed. The proposed preparation strategy is shown in Scheme 1.

\section{Experimental}

2.1. Synthesis of $M I L-53(\mathrm{Fe})$. All the reagents are of analytic grade from Beijing Chemical Reagent Company and used as received. The MIL-53(Fe) was synthesized according to our prior report [25]; the mixture of $\mathrm{FeCl}_{3} \cdot 6 \mathrm{H}_{2} \mathrm{O}$, terephthalic acid, $\mathrm{N}, \mathrm{N}^{\prime}$-dimethyl formamide (DMF), and hydrofluoric acid (HF) with molar ratios of $1: 1: 280: 2$ was heated in Teflon-lined autoclaves. In order to investigate the effect of the crystallinity on the BET surface area, the mixtures were heated at $150^{\circ} \mathrm{C}$ for 1 day and 3 days. A yellow powder MIL$53(\mathrm{Fe})$, loaded with DMF inside its pores, was obtained by filtration method. To remove the occluded DMF, the sample was heated at $150^{\circ} \mathrm{C}$ in air atmosphere overnight and then cooled down to room temperature. To further extract the residual traces of DMF, the sample was stirred with a large volume of deionized water $(1.0 \mathrm{~g}$ of MIL-53 in $500 \mathrm{~mL}$ of water) for $4 \sim 6$ hours and was filtered, and then it was dried at room temperature in air atmosphere.

2.2. Synthesis of Iron Oxide. The conventional $\alpha-\mathrm{Fe}_{2} \mathrm{O}_{3}$ nanoparticle was prepared by heating the mixture of $1.0 \mathrm{~mol} / \mathrm{L} \mathrm{Fe}\left(\mathrm{NO}_{3}\right)_{3}$ solution and $2.0 \mathrm{~mol} / \mathrm{L}$ citric acid solution with the molar ratio of $1: 1$ at $90^{\circ} \mathrm{C}$, dropwise adding the glycol of a dispersant into the mixture until it became viscous, and then the mixture was dried at $120^{\circ} \mathrm{C}$ for $12 \mathrm{~h}$ in a crucible to form a gel. Finally, the gel was calcined at $550^{\circ} \mathrm{C}$ for $2 \mathrm{~h}$ to obtain $\mathrm{Fe}_{2} \mathrm{O}_{3}$ powder, with the obtained sample designated as FO-0.

The as-synthesized iron precursors (MIL-53(Fe)) were positioned at the end of the quartz tube. The quartz tube was then mounted in the middle of the tube furnace. A flow of air was introduced into the quartz tube at a rate of $30 \mathrm{~mL} / \mathrm{min}$ and was heated to $400^{\circ} \mathrm{C}$ for $5 \mathrm{~h}$ at a rate of $10^{\circ} \mathrm{C} / \mathrm{min}$. Then, the system was allowed to cool naturally to room temperature. The two $\alpha-\mathrm{Fe}_{2} \mathrm{O}_{3}$ samples were then 
collected designated as FO- 1 and FO-2, corresponding to the reaction time of 1 day and 3 days, respectively.

2.3. Synthesis of Iron Nitride. The as-synthesized iron precursors (MIL-53(Fe), FO-0 and FO-2) were placed in a quartz tube and treated in a flow of nitrogen $(30 \mathrm{~mL} / \mathrm{min})$ at $150^{\circ} \mathrm{C}$ to remove air in the system. Then, the precursors were heated in $\mathrm{NH}_{3}$ flow $(60 \mathrm{~mL} / \mathrm{min})$ at a rate of $10^{\circ} \mathrm{C} \mathrm{min}^{-1}$ to $500^{\circ} \mathrm{C}$ for $10 \mathrm{~h}$. After that, the sample was cooled naturally to room temperature, then flowing $1 \% \mathrm{O}_{2} / \mathrm{N}_{2}$ for passivation treatment to sample surface. The products were denoted as FN-1, FN-2, FN-3, and FN-4, corresponding to MIL-53(Fe)'s with the different reaction time of 1 day and 3 days and the different iron precursors of FO-0 and FO-2, respectively.

2.4. Characterization Techniques. X-ray diffraction (XRD) measurements were performed using a Rigaku D/MAX-2500 $\mathrm{X}$-ray diffractometer with $\mathrm{CuK} \alpha$ radiation. Samples were analyzed over a range of $5-80^{\circ} 2 \theta$ using a step scan mode with a step rate of $4 \% \mathrm{~min}$. Scanning Electron Microscopy (SEM) measurements were performed using a FEI Quanta $200 \mathrm{~F}$ microscope. Nitrogen adsorption experiments of samples were performed on an ASAP 2020 micropore analyzer. The MIL-53(Fe) samples were evacuated at $150^{\circ} \mathrm{C}$ for $12 \mathrm{~h}$ prior to exposing them to nitrogen gas, while the other samples were evacuated at $300^{\circ} \mathrm{C}$ for $12 \mathrm{~h}$. Their surface areas were calculated using the BET equation and the volumes were determined using the $\mathrm{BJH}$ method.

\section{Results and Discussion}

3.1. XRD Patterns. To probe the effect of MOFs crystallinity on the preparation strategies, we had synthesized MIL-53(Fe) with different crystallization time. As shown in Figure 1, the XRD patterns of MIL-53(Fe), synthesized with different crystallization time, were similar with our prior report [24]. Relative crystallinity was calculated based on the integrated areas of the MIL-53(Fe) characteristic XRD diffraction peaks at $2 \theta=9.26^{\circ}, 11.1^{\circ}, 16.38^{\circ}, 17.2^{\circ}, 18.6^{\circ}$, and $22.3^{\circ}$ using Origin software after baseline correction. By calculation, the MIL$53(\mathrm{Fe})$, with shortened crystallization time, was provided with the relative crystallinity of $82 \%$.

The samples of MIL-53(Fe) with different crystallization time were calcined to obtain $\mathrm{Fe}_{2} \mathrm{O}_{3}$ powders; their XRD patterns are displayed in Figure 2. As shown in Figure 2, the XRD patterns of FO- 1 and FO- 2 were similar with that of FO0 and all the diffraction peaks were able to be assigned to $\alpha$ $\mathrm{Fe}_{2} \mathrm{O}_{3}$ with a rhombohedral phase (JCPDS card number 330064). Also, the results suggested that the hematite could be prepared from the iron-based metal organic framework (FeMOF) serving as reactive hard templates. Compared with the relative crystallinity of FO-1 and FO-2, fewer differences than those of MIL-53 used as hard templates existed. The result indicated that MIL-53 possessed little effects on the material crystallinity.

After nitriding, the FN-3 and FN-4 samples from the $\alpha$ $\mathrm{Fe}_{2} \mathrm{O}_{3}$ precursors showed polycrystalline mixtures consisting mainly of $\alpha-\mathrm{Fe}_{2-3} \mathrm{~N}$ and $\gamma^{\prime}-\mathrm{Fe}_{4} \mathrm{~N}$ (Figures 3(c) and 3(d)).

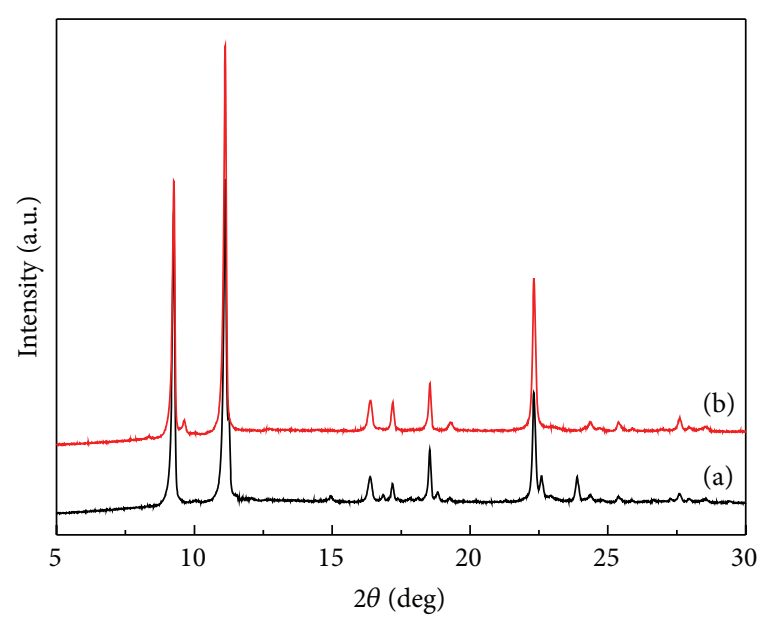

FIgURE 1: XRD patterns of MIL-53 (Fe) samples with different crystallization time. (a) 1 day, (b) 3 days.

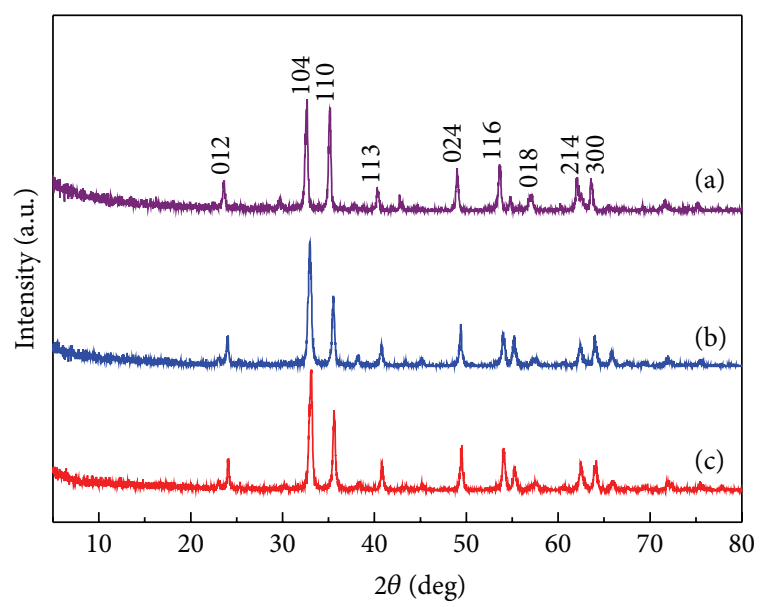

FIGURE 2: XRD patterns of the iron oxide samples. (a) FO-0, (b) FO1 , and (c) FO-2.

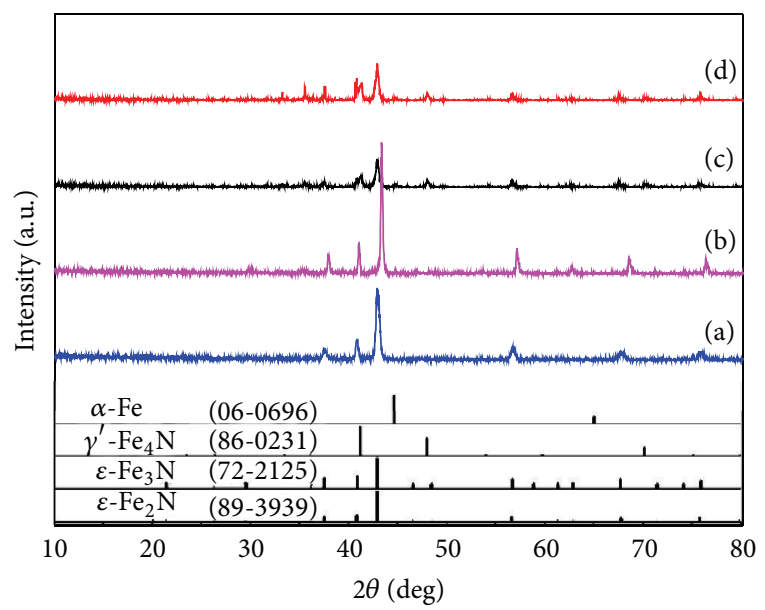

FIGURE 3: XRD patterns of the iron nitride samples. (a) FN-1, (b) FN-2, (c) FN-3, and (d) FN-4. 


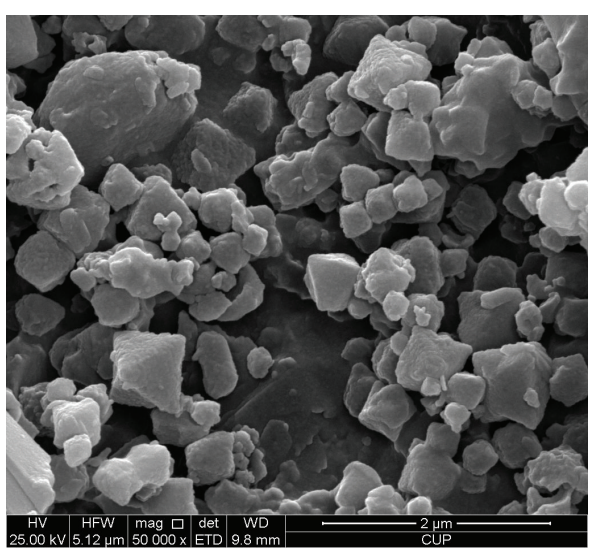

(a)

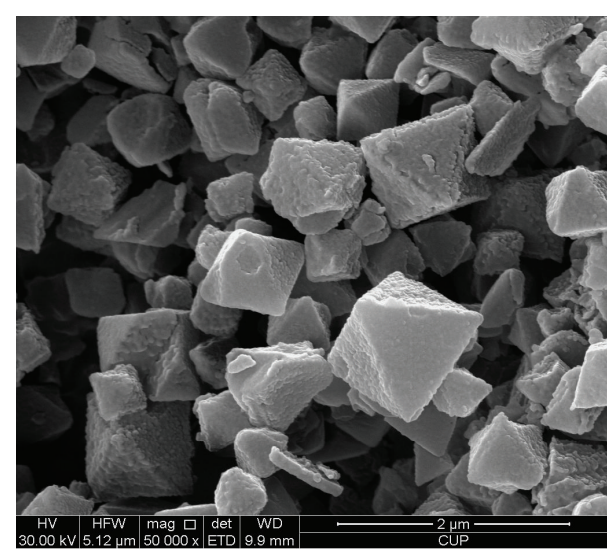

(b)

Figure 4: SEM images of MIL-53 (Fe) samples. (a) 1 day, (b) 3 days.

However, the FN-1 and FN-2 samples all produced $\alpha-\mathrm{Fe}_{2-3} \mathrm{~N}$; almost no peaks related to $\gamma^{\prime}-\mathrm{Fe}_{4} \mathrm{~N}$ were observed. Usually, iron first undergoes initial nitriding to $\mathrm{Fe}_{4} \mathrm{~N}$, and a further nitridation occurs to form $\mathrm{Fe}_{2-3} \mathrm{~N}$ (or $\mathrm{Fe}_{3} \mathrm{~N}_{1+x}$ ) and $\mathrm{Fe}_{2} \mathrm{~N}$. Here, given the same nitriding conditions, MIL-53(Fe) underwent further nitridation to form $\alpha-\mathrm{Fe}_{2-3} \mathrm{~N}$. It was mainly attributed to MIL-53(Fe)'s 3D open microporous system facilitating the contact of $\mathrm{NH}_{3}$. Compared with $\mathrm{FN}-1$ and $\mathrm{FN}-$ 2 , the better the crystallinity of MIL-53(Fe) was, the better the crystallinity of the resulting $\alpha-\mathrm{Fe}_{2-3} \mathrm{~N}$ was. The results presented that well-crystallized MIL-53(Fe) was necessary for the formation of iron nitride with high crystallinity.

3.2. SEM Images. SEM images given in Figure 4 showed that the MIL-53(Fe) sample possessed an octahedral morphology; its content and uniformity varied depending on the material crystallinity. The low-crystallinity samples prepared by shortening the crystallization time exhibited some irregular blocks, which were unformed crystals and unreacted raw materials. Further increasing crystallization time may promote irregular particles evolving into clear-cut submicronsized MIL-53 octahedral crystals.

The morphologies of the oxidized products are shown in Figure 5. The conventional FO-0 (Figure 5(a)) showed compact monodisperse nanospheres with the diameter of approximately $50 \mathrm{~nm}$. However, the other samples from MIL53(Fe) (Figure 5(b)) presented loosely aggregated particles. Among them, FO-1 prepared from MIL-53(Fe) with low crystallinity exhibited irregular particles with the diameters of about $150 \mathrm{~nm}$. Interestingly, the FO-2 sample (Figure 5(c)) from well-crystallized MIL-53(Fe) exhibited octahedral crystals with the size of about $700 \mathrm{~nm}$, faithfully retaining the octahedral morphology of MIL-53(Fe). It demonstrated that the high crystallinity of MIL-53(Fe) was necessary for the formation of $\alpha-\mathrm{Fe}_{2} \mathrm{O}_{3}$ with a regular octahedral morphology. Although a variety of $\alpha-\mathrm{Fe}_{2} \mathrm{O}_{3}$ morphologies were reported in some literatures, the octahedral morphology herein, as far as we know, is the first example. Also, the iron oxide with the octahedral morphology was creatively employed to prepare iron nitride.
In this work, the morphologies of iron nitrides prepared by different strategies are given in Figure 6. The samples from MIL-53(Fe) (Figures 6(a) and 6(b)) showed loosely aggregated particles with rich interparticle porosities. The iron nitride $\mathrm{FN}-3$ corresponding $\alpha-\mathrm{Fe}_{2} \mathrm{O}_{3}$ had compactly aggregated particles. Interestingly, the $\mathrm{FN}-4$ samples from MIL-53 oxide produced peculiar coralloid iron nitrides (Figure 6(d)). It could be because the nitriding temperature led to the disrupting of the MIL-53(Fe) framework; the octahedral morphology disappeared in iron nitride images.

3.3. $\mathrm{N}_{2}$ Adsorption Isotherms. The textural properties and the isotherms of samples were characterized by the $\mathrm{N}_{2}$ adsorption isotherms. $\mathrm{N}_{2}$ adsorption results (Figure 7 and Table 1) showed that the shortened crystallization MIL-53 sample had a BET surface area of $611 \mathrm{~m}^{2} \cdot \mathrm{g}^{-1}$. The surface area was lower than $1031 \mathrm{~m}^{2} \cdot \mathrm{g}^{-1}$ for the completing crystallized sample. Similarly, Langmuir surface areas, pore volumes, and pore diameter reduced due to some raw materials blocking the pore. Also, the results (see Figure 8 and Table 1) exhibited that the FO-1 and FO-2 samples from MIL-53 had larger BET surface area and total pore volume than those of the conventional FO-0 sample using sol-gel method. Such higher surface areas for both were believed to benefit from the calcination of the MOFs template. Compared to the FO-1 sample, the FO-2 sample had a relatively higher BET surface of $170 \mathrm{~m}^{2} \cdot \mathrm{g}^{-1}$. It illustrated that FO-2 sample was retaining not only the octahedral morphology of MIL-53(Fe) but also the microporous framework. The result was consistent with the SEM image result. As a result, it may be concluded that the crystallinity and BET surface of MOFs template had significant effects on the pore properties of iron oxide.

In addition, in accordance with these observed morphologies, nitrogen adsorption experiment results (see Figure 9 and Table 1) determined that the BET specific area of the sample FN-0 4 was $21,41,12$, and $17 \mathrm{~m}^{2} \cdot \mathrm{g}^{-1}$, respectively. The surface areas of the $\mathrm{FN}-1$ and $\mathrm{FN}-2$ samples prepared from MIL-53(Fe) were larger than those of FN3 and $\mathrm{FN}-4$ using $\alpha-\mathrm{Fe}_{2} \mathrm{O}_{3}$ as the precursor. Although 


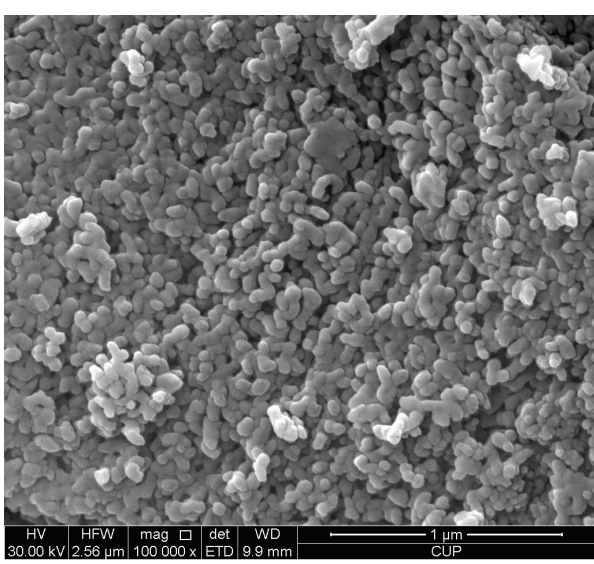

(a)

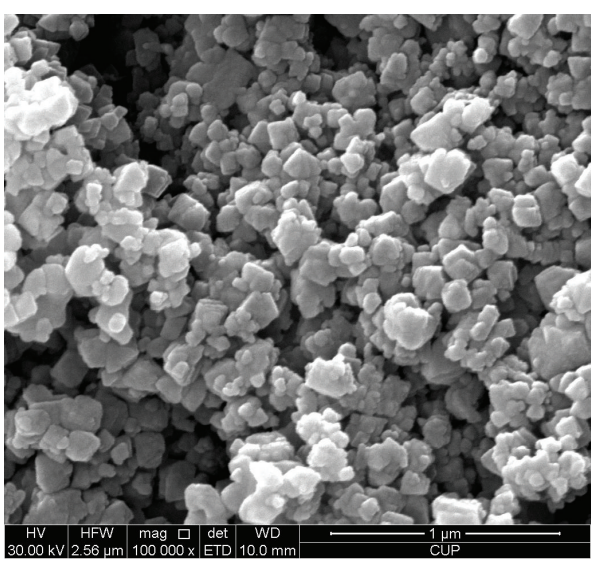

(b)

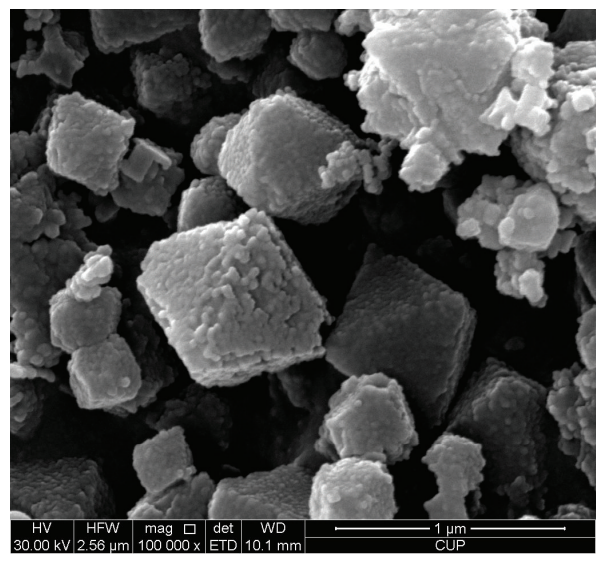

(c)

FIgURE 5: SEM images of the iron oxide samples. (a) FO-0, (b) FO-1, and (c) FO-2.

TABLE 1: Textural properties of samples.

\begin{tabular}{lcccc}
\hline Samples & $S_{\text {BET }} /\left(\mathrm{m}^{2} \cdot \mathrm{g}^{-1}\right)$ & $S_{\text {Langmuir }} /\left(\mathrm{m}^{2} \cdot \mathrm{g}^{-1}\right)$ & Pore volume $/\left(\mathrm{cm}^{3} \cdot \mathrm{g}^{-1}\right)$ & Pore size $/ \mathrm{nm}^{-1}$ \\
\hline MIL-53-a & 611 & 854 & 0.39 & 0.47 \\
MIL-53-b & 1031 & 1286 & 0.19 & 1.85 \\
FO-0 & 68 & 79 & 0.20 & 1.12 \\
FO-1 & 106 & 118 & 0.28 & 1.7 \\
FO-2 & 170 & 183 & 0.07 & 2.18 \\
FN-1 & 21 & 27 & 0.12 & 2.13 \\
FN-2 & 41 & 46 & 0.02 & 3.85 \\
FN-3 & 12 & 18 & 0.05 & 1.5 \\
FN-4 & 17 & 20 & & 1.93 \\
\hline
\end{tabular}

the nitridation reaction finally disrupted the MIL-53(Fe) framework through the decomposition of dicarboxylate units, the initial microporous crystalline framework was beneficial for the formation of interparticle porosities. Accordingly, MIL-53(Fe) was considered as a hard template to impart a certain degree of porosity to the iron nitride product. Apparently, the increasing of BET surface area for iron nitrides from MIL-53 raw material was mainly ascribed to the prior nitriding pathway.

\section{Conclusions}

In this work, the iron nitride was creatively prepared through thermal ammonia nitridation of the iron-based metal organic framework (Fe-MOF) serving as the reactive hard template and the iron source. The results demonstrated that the iron nitride directly prepared from the MIL-53(Fe) was provided with the optimal surface area in all the preparation strategies. The high surface area was attributed to the formation of 


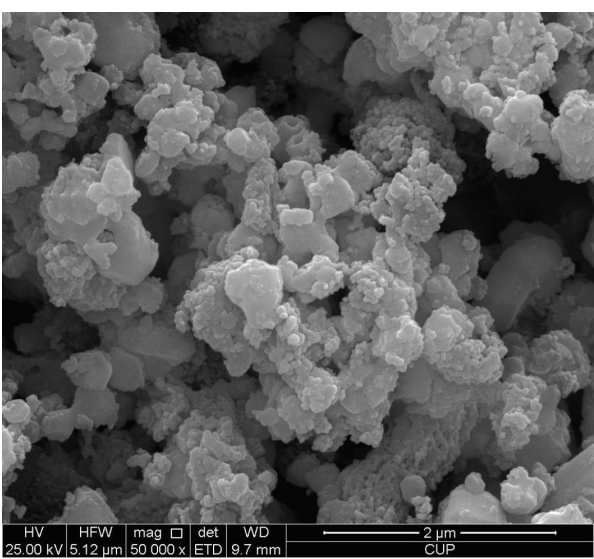

(a)

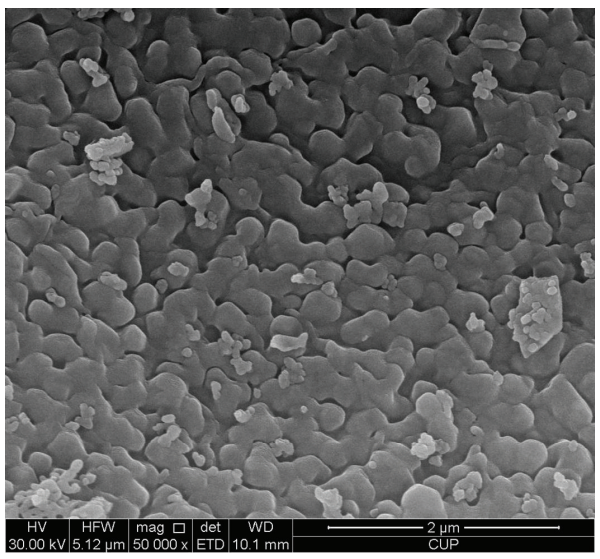

(c)

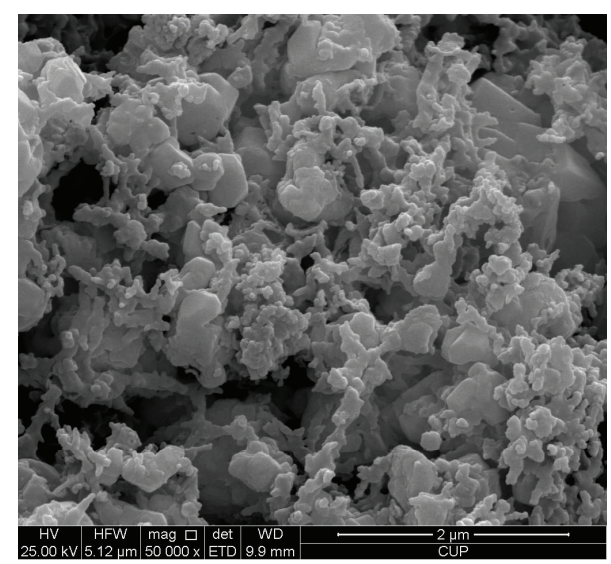

(b)

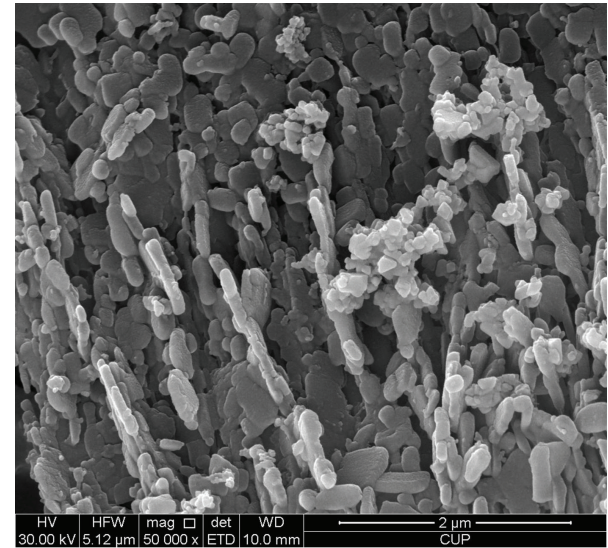

(d)

FIGURE 6: SEM images of iron nitride samples. (a) FN-1, (b) FN-2, (c) FN-3, and (d) FN-4.

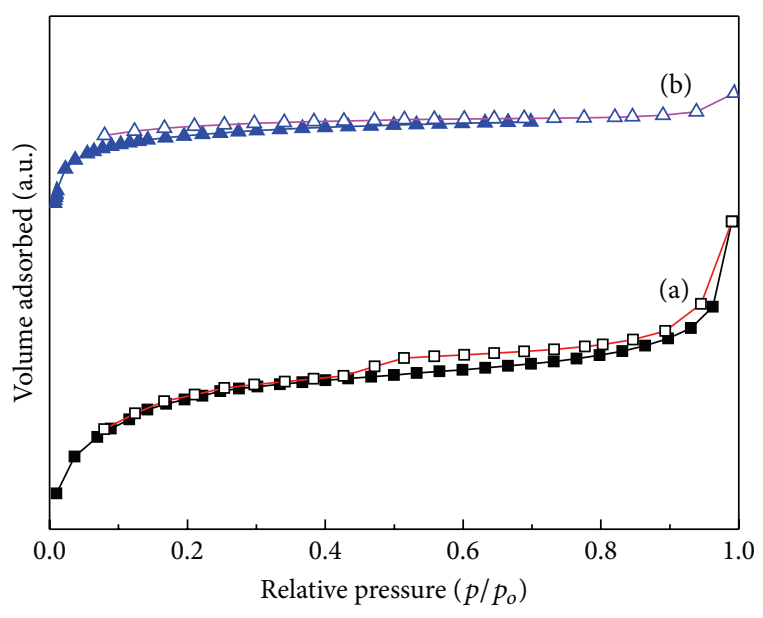

FIGURE 7: $\mathrm{N}_{2}$ adsorption isotherms of MIL-53 (Fe) samples with different crystallization time. (a) 1 day, (b) 3 days.

interparticle porosity benefited from the initial microporous crystalline framework of the MIL-53(Fe). Accordingly, the MIL-53(Fe) was considered as the hard template to impart a certain degree of porosity to the iron nitride. Thus, the

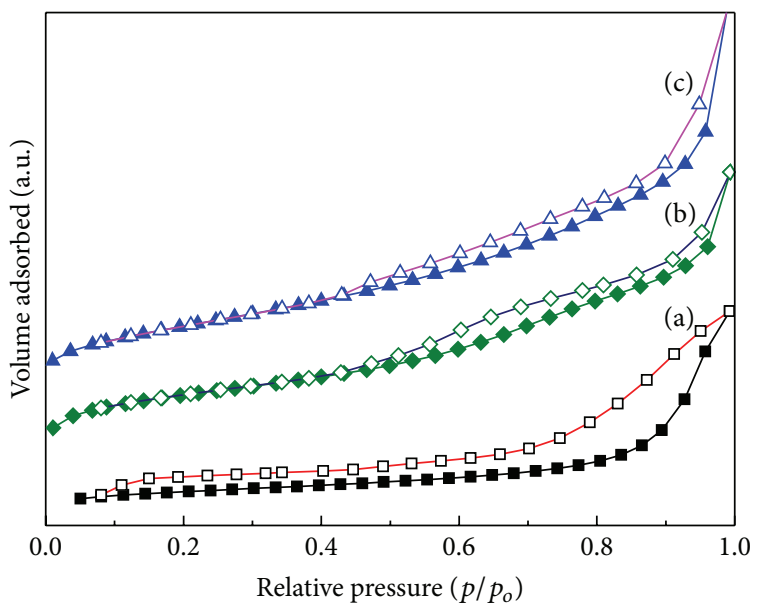

FIGURE 8: $\mathrm{N}_{2}$ adsorption isotherms of the iron oxide samples. (a) FO-0, (b) FO-1, and (c) FO-2.

increasing of BET surface area for iron nitrides from MIL53 raw material was mainly ascribed to the prior nitriding pathway. In addition, this new synthetic strategy is able to become a general approach for the preparation of late transition metal nitrides with peculiar properties. 


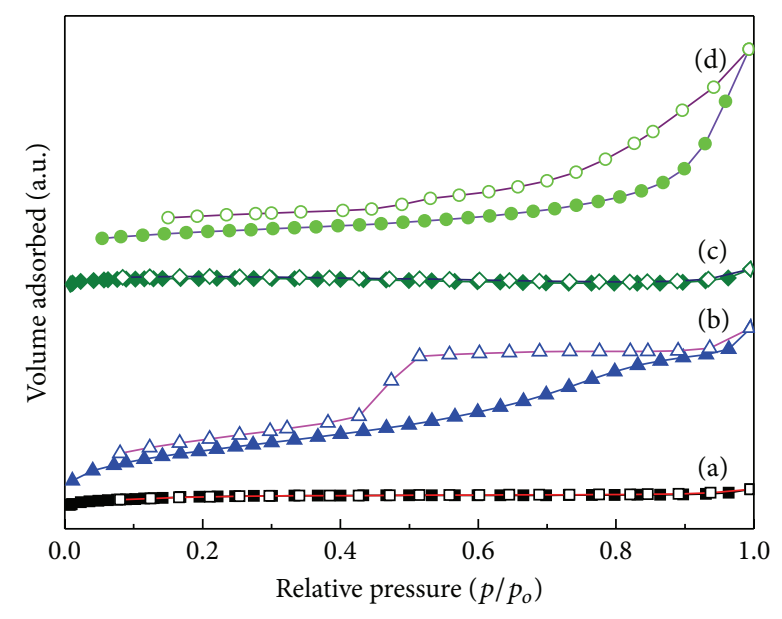

FIGURE 9: $\mathrm{N}_{2}$ adsorption isotherms of the iron nitride samples. (a) FN-1, (b) FN-2, (c) FN-3, and (d) FN-4.

\section{Conflict of Interests}

The authors declare that there is no conflict of interests regarding the publication of this paper.

\section{Acknowledgment}

The authors acknowledge the support of this work by the National Natural Science Foundation of China (Grant nos. 21106123 and 21303157).

\section{References}

[1] S. T. Oyama, The Chemistry of Transition Metal Carbides and Nitrides, Blackie Academic Professional, Glasgow, UK, 1996.

[2] R. W. Chorley and P. W. Lednor, "Synthetic routes to high surface area non-oxide materials," Advanced Materials, vol. 3, no. 10, pp. 474-485, 1991.

[3] L. Volpe and M. Boudart, "Compounds of molybdenum and tungsten with high specific surface area: I. Nitrides," Journal of Solid State Chemistry, vol. 59, no. 3, pp. 332-347, 1985.

[4] C. H. Jaggers, J. N. Michaels, and A. M. Stacy, "Preparation of high-surface-area transition-metal nitrides: molybdenum nitrides, $\mathrm{Mo}_{2} \mathrm{~N}$ and MoN," Chemistry of Materials, vol. 2, no. 2, pp. 150-157, 1990.

[5] J. B. Claridge, A. P. E. York, A. J. Brungs, and M. L. H. Green, "Study of the temperature-programmed reaction synthesis of early transition metal carbide and nitride catalyst materials from oxide precursors," Chemistry of Materials, vol. 12, no. 1, pp. $132-142,2000$.

[6] X. Z. Chen, J. L. Dye, H. A. Eick, S. H. Elder, and K.-L. Tsai, "Synthesis of transition-metal nitrides from nanoscale metal particles prepared by homogeneous reduction of metal halides with an alkalide," Chemistry of Materials, vol. 9, no. 5, pp. 11721176, 1997.

[7] M. A. Sriram, P. N. Kumta, and E. I. Ko, "Interaction of solvent and the nature of adducts on the chemical synthesis of molybdenum nitride powders," Chemistry of Materials, vol. 7, no. 5, pp. 859-864, 1995.
[8] J. Choi and E. G. Gillan, "Solvothermal metal azide decomposition routes to nanocrystalline metastable nickel, iron, and manganese nitrides," Inorganic Chemistry, vol. 48, no. 10, pp. 4470-4477, 2009.

[9] A. Fischer, J. O. Müller, M. Antonietti, and A. Thomas, "Synthesis of ternary metal nitride nanoparticles using mesoporous carbon nitride as reactive template," ACS Nano, vol. 2, no. 12, pp. 2489-2496, 2008.

[10] C. Giordano, C. Erpen, W. Yao, B. Milke, and M. Antonietti, "Metal nitride and metal carbide nanoparticles by a soft urea pathway," Chemistry of Materials, vol. 21, no. 21, pp. 5136-5144, 2009.

[11] M. Yang and F. J. DiSalvo, "Template-free synthesis of mesoporous transition metal nitride materials from ternary cadmium transition metal oxides," Chemistry of Materials, vol. 24, no. 22, pp. 4406-4409, 2012.

[12] Y. Koltypin, X. Cao, R. Prozorov, J. Balogh, D. Kaptas, and A. Gedanken, "Sonochemical synthesis of iron nitride nanoparticles," Journal of Materials Chemistry, vol. 7, no. 12, pp. 24532456, 1997.

[13] K. Nishimaki, S. Ohmae, T. A. Yamamoto, and M. Katsura, "Formation of iron-nitrides by the reaction of iron nanoparticles with a stream of ammonia," Nanostructured Materials, vol. 12, no. 1, pp. 527-530, 1999.

[14] Y. Han, H. Wang, M. Zhang, M. Su, W. Li, and K. Tao, "Low-temperature approach to synthesize iron nitride from amorphous iron," Inorganic Chemistry, vol. 47, no. 4, pp. 1261$1263,2008$.

[15] M. Zheng, X. Chen, R. Cheng et al., "Catalytic decomposition of hydrazine on iron nitride catalysts," Catalysis Communications, vol. 7, no. 3, pp. 187-191, 2006.

[16] D. Moszyński, K. Kiełbasa, and W. Arabczyk, "Influence of crystallites' size on iron nitriding and reduction of iron nitrides in nanocrystalline Fe-N system," Materials Chemistry and Physics, vol. 141, no. 2-3, pp. 674-679, 2013.

[17] M. O'Keeffe, "Design of MOFs and intellectual content in reticular chemistry: a personal view," Chemical Society Reviews, vol. 38, no. 5, pp. 1215-1217, 2009.

[18] B. Liu, H. Shioyama, T. Akita, and Q. Xu, "Metal-organic framework as a template for porous carbon synthesis," Journal of the American Chemical Society, vol. 130, no. 16, pp. 5390-5391, 2008.

[19] R. K. Bhakta, J. L. Herberg, B. Jacobs et al., "Metal—organic frameworks as templates for nanoscale $\mathrm{NaAIH}_{4}$," Journal of the American Chemical Society, vol. 131, no. 37, pp. 13198-13199, 2009.

[20] F. Schröder, D. Esken, M. Cokoja et al., "Ruthenium nanoparticles inside porous $\left[\mathrm{Zn}_{4} \mathrm{O}(\mathrm{bdc})_{3}\right]$ by hydrogenolysis of adsorbed $[\mathrm{Ru}(\operatorname{cod})(\mathrm{cot})]$ : a solid-state reference system for surfactantstabilized ruthenium colloids," Journal of the American Chemical Society, vol. 130, no. 19, pp. 6119-6130, 2008.

[21] S. Hermes, F. Schrder, S. Amirjalayer, R. Schmid, and R. A. Fischer, "Loading of porous metal-organic open frameworks with organometallic CVD precursors: inclusion compounds of the type $\left[\mathrm{L}_{n} \mathrm{M}\right]_{a} @ \mathrm{MOF}-5$," Journal of Materials Chemistry, vol. 16, no. 25, pp. 2464-2472, 2006.

[22] J. Kim, G. T. Neumann, N. D. McNamara, and J. C. Hicks, "Exceptional control of catalytic hierarchical carbon supported transition metal nanoparticles using metal-organic framework templates," Journal of Materials Chemistry A, vol. 2, no. 34, pp. 14014-14027, 2014. 
[23] P. L. Llewellyn, P. Horcajada, G. Maurin et al., "Complex adsorption of short linear alkanes in the flexible metal-organicframework MIL-53(Fe)," Journal of the American Chemical Society, vol. 131, no. 36, pp. 13002-13008, 2009.

[24] W. Cho, S. Park, and M. Oh, "Coordination polymer nanorods of Fe-MIL-88B and their utilization for selective preparation of hematite and magnetite nanorods," Chemical Communications, vol. 47, no. 14, pp. 4138-4140, 2011.

[25] S. Y. Liu, Y. Zhang, Y. Meng, F. Gao, S. J. Jiao, and Y. C. Ke, "Fast syntheses of MOFs using nanosized zeolite crystal seeds in situ generated from microsized zeolites," Crystal Growth \& Design, vol. 13, no. 7, pp. 2697-2702, 2013. 

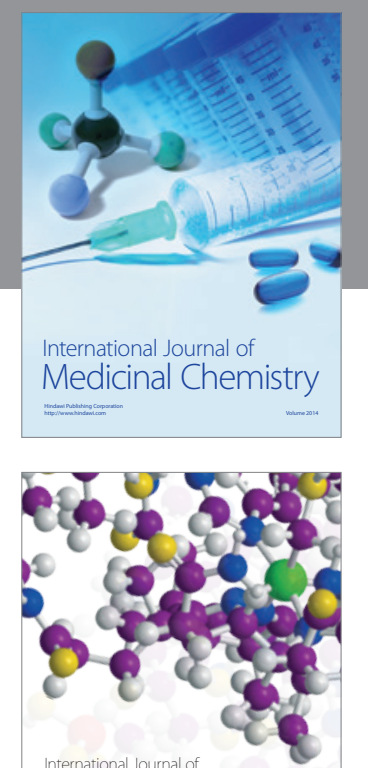

\section{Carbohydrate} Chemistry

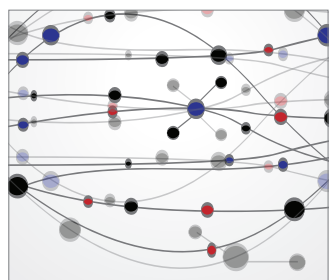

The Scientific World Journal
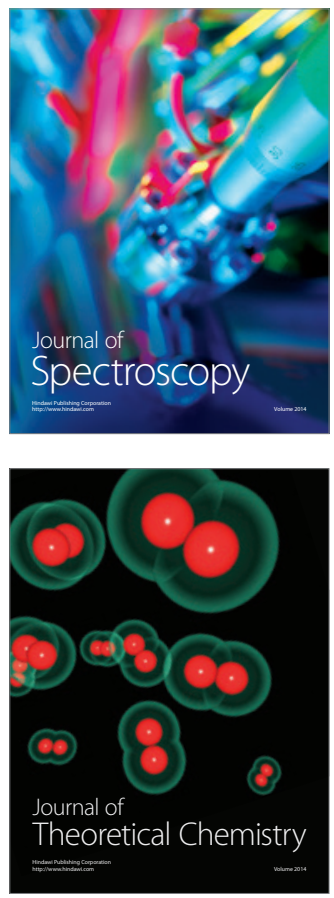
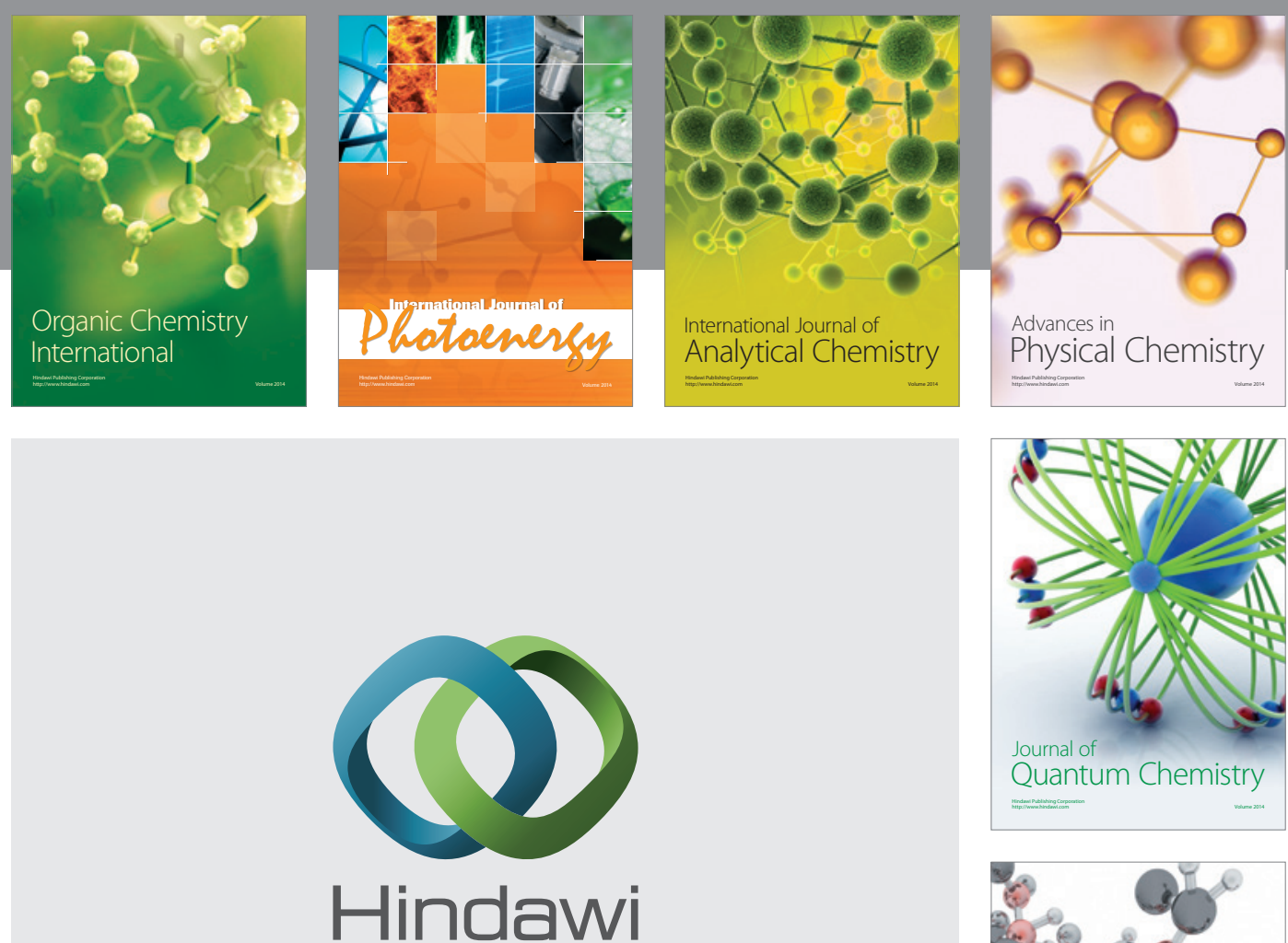

Submit your manuscripts at

http://www.hindawi.com

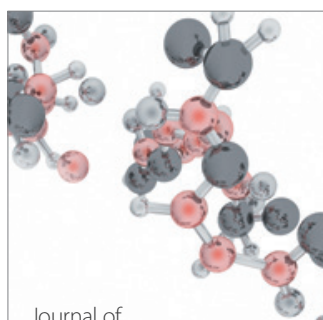

Analytical Methods

in Chemistry

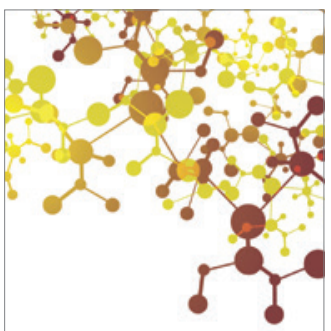

Journal of

Applied Chemistry

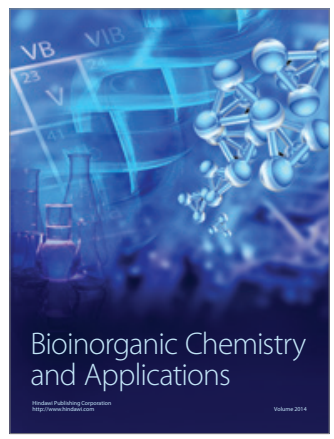

Inorganic Chemistry
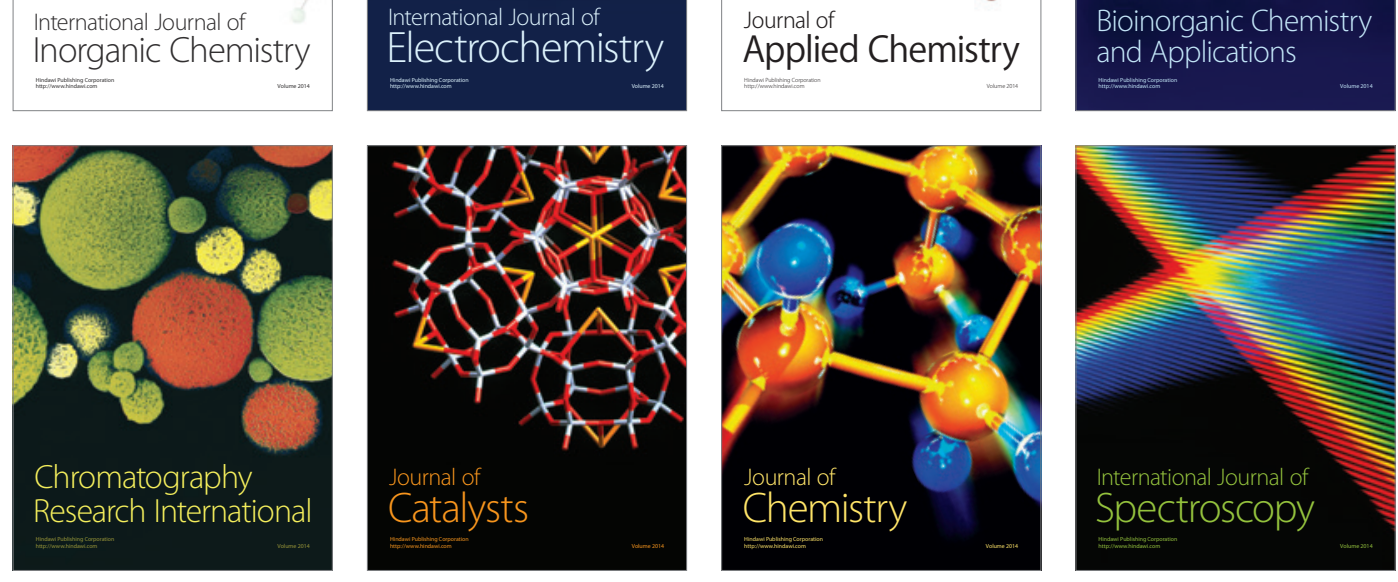